\title{
Cloud Computing Technology for library Services a big boon
}

\author{
Pankaj Bhagat
}

\begin{abstract}
Cloud computing technology, service and application that are similar to internet and converts into the independent utility. Impediment on cloud computing for instance sparing arrangement creative improvement have not only affected simply the association \& subtleties of factsyet what's in addition reference organizations gave by the libraries. Libraries and their assets have to some degree moved to an e-world. From now on library clients can get to our assets from outside the physical library through their pc. These advancement can be generally gotten anyway ought to endure various changes by infers where they are offered by the libraries. Taking up of cloud computing is not a simple task for libraries. The inclination/ detriment clarification and its proposition must be truly viewed as beforehand putting essential data. The essential thought in this paper is to evaluate cloud benefits for the library. This study investigate the application of cloud computing and its utilities for library the executives. Cloud application gives its administrations on solicitation to its clients. Data aggregation is among indispensable administration given by cloud computing. It has the data on their server and user can get services from these servers. Data providers and servers are diverse characters the perspective of data aggregating raises various security challenges. A free part is required to guarantee that data is unequivocally encouraged in to the distributed storage server. In this paper we will examine the unlike frameworks that are utilize protected data storing on cloud. Cloud computing data amassing distributed storage server.
\end{abstract}

Keywords: Cloud computing, Data Storage, Cloud storage space

\section{I.INTRODUCTION}

Presentation imaginative enhancements have impacted not simply the association and inception of information yet moreover the reference organizations gave by the libraries. Libraries and their advantages have to some degree moved to an e-world. Hence library clients can get to administrations from outside the physical library. These advancement can be commonly gotten have experienced various changes offered by the libraries. Selection of cloud computing was not a basic assignment for libraries. The inclinations/inconveniences highlights and its proposals must be truly viewed as already putting data on the cloud. The critical thought in this paper is to evaluate cloud administrations for the library. This paper gives compact information on distributed computing and its application for library organizations with a bizarre reference to the makers information centre. With the guide of the distributed computing the client is approved to approach all the more capably utilize the applications by the more administrations stipend memory and focal stockpiling. there are number of critical advantages of the distributed computing in the library the library can be digitalized all library

Revised Manuscript Received on November 05, 2019.

Pankaj Bhagat, Ph.D. (Pursu.), Madhav University, Dist. Sirohi, Mount Abu, Rajasthan-307026 housekeeping capacities can be automated. The word cloud is used as an image for the web in perspective on the systematized use of a cloud-like shape to connote a framework on correspondence schematics and later to differentiate the web in pc sort out diagrams as a thought of the shrouded structure it addresses. The cloud picture was used to address the web as on schedule as 1994. Circulated figuring is the use of preparing resources hardware and programming that are passed on as a help over network usually the web the name starts from the usage of a cloud-shaped picture as a thought for the astounding structure it contains in system diagrams. Cloud enrolling depends remote organizations with a customer's data programming and count. cloud figuring is a web based enrolling where shared resources applications and information are given to the course of action of pcs and various contraptions on demand using web technology. Therefore the system of conveyed registering is being done through game plan of web engaged applications stacked on the server with genuine access rights. To encourage the library administrations number of utilizations and administrations are conveyed through web by the distributed computing. Cloud computing is a specific sort of registering which is relied upon shared figuring assets.

\section{THERE ARE FOUR RULE SORTS OF DISTRIBUTED STORAGE}

* Singular distributed storage: - it is generally called convenient distributed storage. In this sort storing people data is taken care of in the cloud and he/she may get to the data from wherever.

* Open distributed storage: - without trying to hide distributed storage the undertaking and limit authority centre are secluded and there aren't any cloud resources set away in the endeavours server ranch. The distributed storage provider totally manages the endeavours open distributed storage.

* $\quad$ Private distributed storage:- in private distributed storage the undertaking and distributed storage provider are fused in the endeavours server ranch. In private distributed storage the limit provider has establishment in the undertakings server ranch that is conventionally directed by the limit provider.

* Distribution: - helps settle the potential for security and execution concerns while up 'til now offering the advantages of distributed storage. Cream distributed storage: it is a mix of open and private distributed storage where some fundamental data abides in the endeavours 
private cloud while other data is taken care of and accessible from an open distributed storage provider.

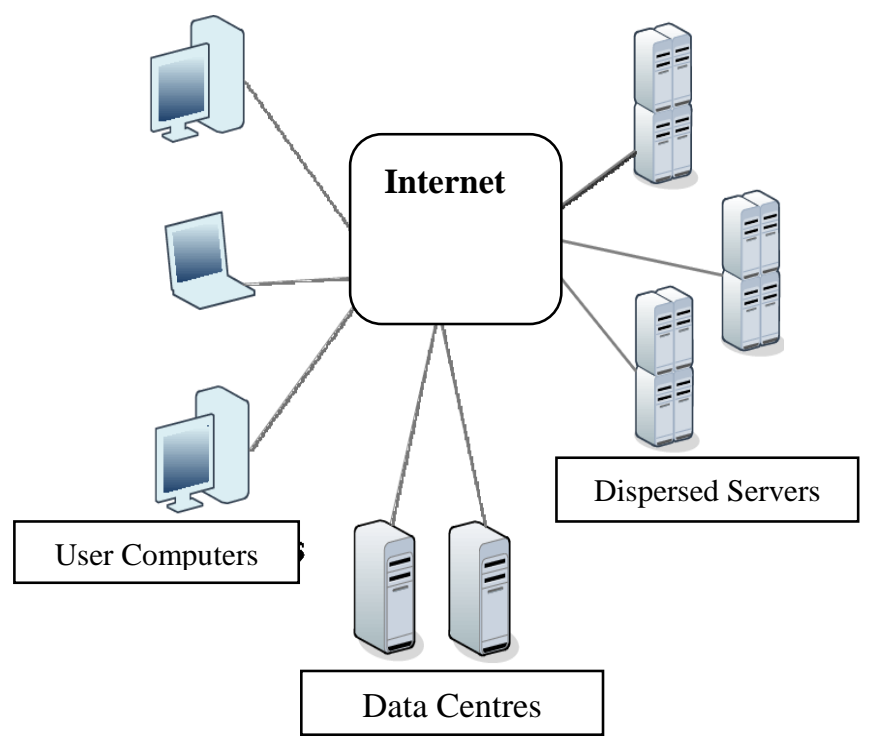

Working of the cloud computing, figure: 1

\section{PARTS OF CLOUD COMPUTING}

Cloud computing comprises of different parts. Every one of these parts must be streamlined for a verified and well-working application for distributed computing.

\section{FUNCTIONS}

The administration is regularly thought as the application. In spite of the fact that it is somewhat right given the reality that it gives the capacities, the application is totally unique since it is through the application that the administration is figured it out. This is the place the product engineers need to centre in terms of guaranteeing the application will function true to form. Streamlining of the application is based on the genuine coding of designers. Through broad testing on load taking care of, security and usefulness, the application can fill in true to form.

\section{HOW TO RUN}

In customary sites or applications that don't manage distributed computing, the application is straightforwardly associated with the server. In cloud computing, the application is still propelled to another application called the Platform. The stage generally comes as the programming language such as Ajax (Asynchronous JavaScript and XML) or Ruby on Rails. Now, the individuals who select to look for distributed computing suppliers should pursue the set programming dialects Amazon EC2-Virtual IT, Google App Engine, Microsoft Office Online (SaaS),Apple i cloud (Network storage),Digital Ocean (Iaas/Paas)

\section{HOW TO STORE}

Everything that the application knows and the capacities that could be given by the administration are conceivable through capacity. The capacity holds relevant information and data on how they will be implemented. Optimization on capacity depends on how the storeroom is shielded from various assaults and the accessibility of back-up. Could Computing is consistently about consistency and accessibility of administration which will normally require the capacity to be accessible constantly.

\section{VII.COMPONENTS OF CLOUD STORAGE}

Each capacity, administration and the capacity of capacity to give the required information is as it were conceivable through enhanced framework. This could be considered as the stage behind the capacity as the foundation enables the capacity to manage load issues. The framework is a stage wherein it gauges the capacity of the capacity against the quantity of solicitations. The framework can roll out certain improvements by load adjusting and smooth administration

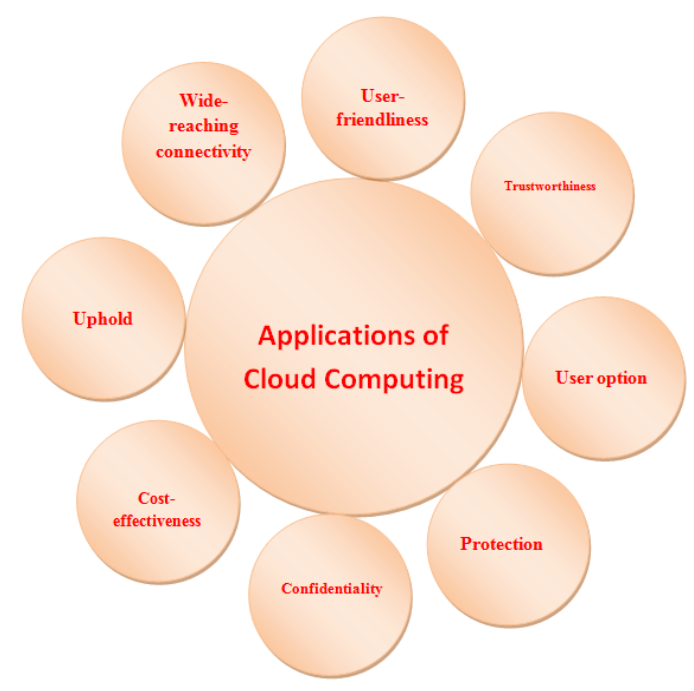

Benefits cloud computing, figure: 2

\section{APPLICATION OF CLOUD COMPUTING IN LIBRARIES}

Libraries, as moving their administrations with the connection of cloud and systems administration encourage getting to these administrations anyplace and whenever. In the libraries the accompanying potential territories were recognized where distributed computing administrations and applications might be applied in following:

* Building Digital Repositories-In the current circumstance, each library needs an advanced library to make their assets data and administrations at an effective level to guarantee get to by means of the systems. Accordingly every library is having an advanced library that created by utilizing any advanced library programming. In association with cloud based computerized library programming, D-space is having two programming projects in particular D space and Fedora house however D-space is generally utilized for building advanced stores. Comparative with Fedora normal. Dura cloud gives register answers for creating advanced libraries with standard interface also, source codes for the both programming. 
Looking through Library Data-Web share the board framework encourages to create on open and cooperative stage in which every library can share their assets, administrations, thoughts and issues with library network on the mists.

On the other hand the primary point of web scale administrations is to give cloud based stages, assets and administrations with money saving advantage also, adequacy to share the information and building the expand joint effort in the network. OCLC is truly outstanding model for utilizing distributed computing for sharing library is information for a considerable length of time together.

* Site Hosting-Website facilitating is perhaps the most punctual reception of distributed computing the same number of associations counting libraries liked to have their site on third get-together specialist organizations as opposed to facilitating and keeping up their claim servers. Google locales servers for instance of an assistance for facilitating site outside of the library's servers and permitting for numerous editors get to the site from shifted areas.

* Looking through Scholarly Content-At present Knimbus proposed a free offer to get registered to enable the libraries for dynamic scanning and furthermore for single point search interface, boost the use of all e-assets, modified pursuit crosswise ovep chose sources, diminishes clamor and features applicable substance and instruments to help the total search lifecycle. As of now Information and library organize (INFLIBNET) focus (http://www.inflibnet.ac.in) the cloud administration into its UGC INFONET Digital Library Consortium so as to look and recover insightful substance connected there in. It is right now utilized in more than 600 scholastic organization and R\&D labs by researcher's specialists and researchers as well as more than 50,000 specialists.

* Record Storage-To get to any documents on the web distributed computing present number of administrations.

* Building Community Power-distributed computing innovation offers incredible open doors for libraries to construct organize, among the libraries and data science experts just as other intrigued individuals including data searchers by utilizing long range informal communication devices. The most well known long range interpersonal communication administrations viz. twitter and face book which assume a key job.

* Building network control- This helpful exertion of libraries will make efficient, efficiencies and more extensive acknowledgment.

\section{Benefits of cloud computing framework in E-Libraries-}

In cloud computing information is spared into cloud conditions. Anything that we are doing on PC likely for even on MS Word application is spared through web.

* It will concern an affordable thing. We can pay in portion premise moreover.
* Limit is expanded as contrast with neighborhood servers and nearby structures. We can store more information and Information.

* Data can be recovered from anyplace and whenever or you can adjust the cloud accessibility through the utilizing of web medium.

* We need not to keep our programming projects and application bundles refreshed or redesigned. It spares parcel within recent memory and furthermore discharged structure permitting issues.

* Clients won't get experience delays while taking a shot at PC application or frameworks.

* Its gave programmed update to specific stage just as for programming, by means of web premise.

Spare the client's time.

Numerous gets to be likewise conceivable with this sort of worldview.

E-Library is an answer for library which is a developing living being.

It can get to all around.

Each individual their book at an equivalent time by means of the asset sharing.

\section{Uses of cloud computing framework in E-libraries-}

One of the main jars of utilizing distributed computing is in the e-libraries web association is must be executed.

In the event that web association is going down it will get difficult to work without web association.

Information you put away on the web is more verified in the cloud condition as contrast with neighborhood machines or frameworks.

Rapid association is required on the off chance that you are perusing huge substance.

Information is a current on the other server there is no immediate control at where your information is really present day.

Clients need to at any rate getting information about the web wordings

11. Highlights of cloud computing:-

There are numerous traits of distributed computing. The first is on-demand self-organization, where a buyer of organizations is given the necessary resources without human intercession and correspondence with cloud provider. The resulting trademark is wide framework get to, which suggests resources can be gotten to from wherever through a standard part by dainty or thick client stages such wireless, workstation, and PC. Resource pooling is another trademark, which infers the advantages are pooled with the ultimate objective for multitenant to share the benefits. In the multi-inhabitant model, resources are consigned capably to a client and after the customer finishes it, it will in general be doled out to another to respond to high resource demand. Notwithstanding whether the advantages are consigned to customers on demand, they don't have the foggiest thought regarding the territory of these consigned resources for a checked and well-working application for circulated figuring.

\section{Function:-}

The organization is habitually thought as the application. Notwithstanding the way that it is not completely right given the truth that it gives the limits, the application is absolutely exceptional considering the 
way that it is through the application that the organization is made sense of it.

This is the spot the item creators need to focus as far as ensuring the application will work exactly as expected. Improvement of the application depends on the genuine coding of designers. Through expansive testing on load dealing with, security and value, the application can work exactly as expected.

\section{Application:-}

In normal locales or applications that don't oversee conveyed processing, the application is direct connected with the server. In cloud computing, the application is still moved to another application called the Platform. The stage by and large comes as the programming language, for example, Ajax (Asynchronous JavaScript and XML) or Ruby on Rails.

Presently, the people who pick to search for circulated registering providers should seek after the set programming lingos that could be run in the stage. But most programming, vernaculars could be pushed in different stages, an unbelievable application with consistent reviving capacity is an outright need for circulated processing.

\section{STORAGE}

Everything that the application knows and the limits that could be given by the organization are possible through limit. The limit holds significant data and information on how they will be implemented. Optimization on limit relies upon how the storeroom is protected from different attacks and the openness of back-up. Could Computing is reliably abou consistency and openness of organization which will typically require the ability to be available always.

\section{CONFIDENTIALITY}

The noteworthy challenge in distributed computing is protection. Data protection suggests getting to the data just by affirmed customers and is immovably related to authentication. In another way grouping infers keeping customer's data secret in the cloud structures. As we are taking care of the data on a remote server and moving the control over the data to the provider here raises the request, for instance, for ensuring grouping, cryptographic encryption computations and strong affirmation segments can be used. Encryption is the path toward changing over the data into a structure called figure message that can be seen extraordinarily by the affirmed customers. Encryption is a capable strategy for verifying the data anyway have the obstruction that data will be lost once the encryption key is steeled. Computations. Blowfish is a fat and clear encryption count.

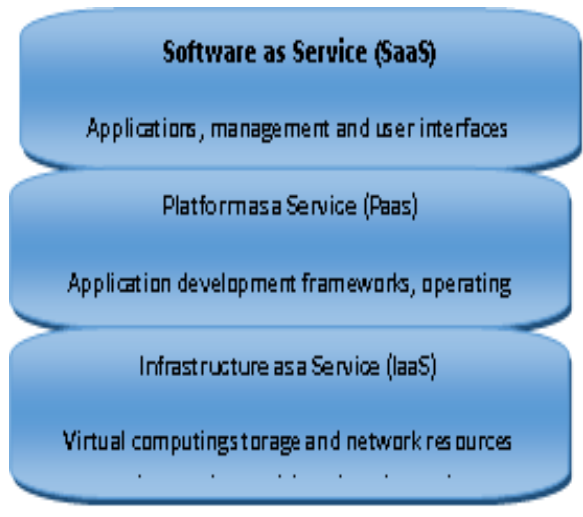

Cloud computing platform, figure: 3

\section{TYPE OF CLOUD COMPUTING}

\section{* Public cloud:-}

This sort of disseminated processing is the ordinary model that everyone thinks about when they imagine circulated figuring. In this model, traders effectively assign resources (hard drive space, RAM, and processor control) on a for every customer premise through web applications. Example are ESD's,eNight cloud, Amazon elastic commute cloud(EC2),IBM's blue cloud, Goggle App engine and windows azure service platform • Unlimited access-As long as you have web get to and an ideal contraption, for instance, a propelled cell phone or Workstation, can get to your data wherever. Unlimited data limit Public conveyed processing is versatile to meet your business 'developing data amassing and dealing with needs.

\section{Hybrid cloud:-}

This model gets business' gear together with dispersed processing. Generally, one of the business applications, for instance, Exchange Server 2007 or Microsoft Dynamics will connect with a dealer encouraged organization. Example of the hybrid computing is Pivotal cloud foundry, Red hat open shift, IBM's blue mix and Append. Hardware: Hybrid conveyed registering requires that you have or purchase gear to speak with the encouraged plan. Software: despite hardware essentials, business ought to have or purchase the item to control and store data.

\section{Private cloud :-}

Generally called "internal cloud computing", Private circulated processing is the individuals to happen to virtualization. While like virtualization at the server, workstation and application levels.

private dispersed registering has redesigned features that interest to various associations like:-

Increased data security-The business' are answerable for security since data never leaves the framework.

Simple consistence execution:-Depending upon the vertical market, government rules may deny business' from using standard or blend conveyed figuring. Private dispersed registering permits to misuse disseminated figuring features while keeping each and every coordinated datum on area and secure.

Customized IT sort out control-By keeping the cloud private, they are permitted to modify their framework to meet their specific business needs.

Appropriated processing with respect to Libraries.

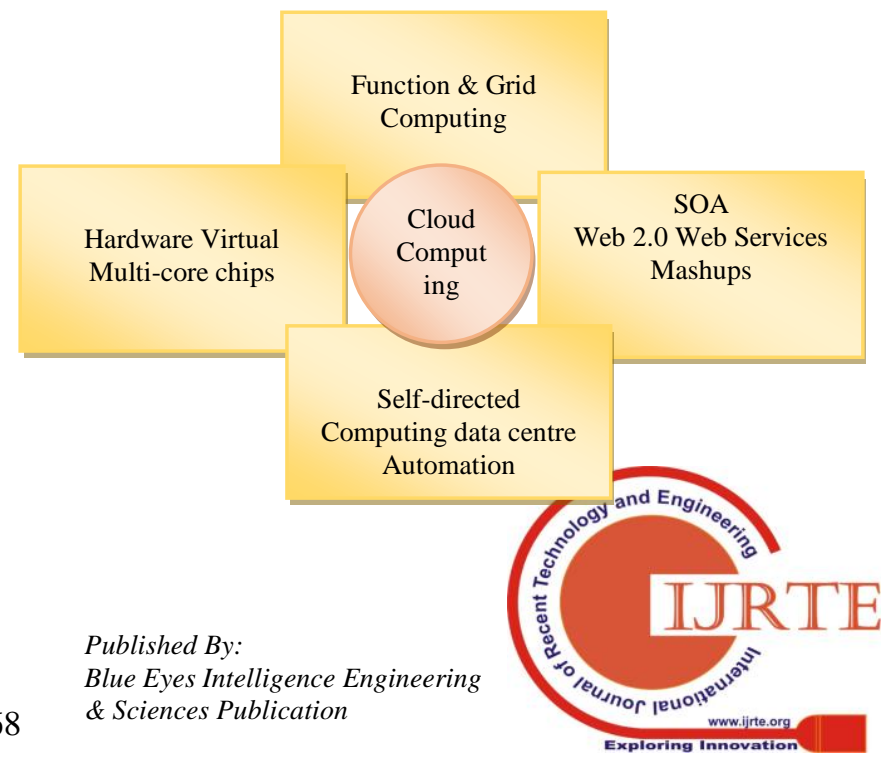


Concept of cloud computing platform, figure: 4

* Libraries are moving towards cloud and endeavouring to give sort out based organizations.

Moving to cloud-based organizations infers, the library housekeeping errands, modernized libraries, etc., are encouraged on cloud-based framework. In the bleeding edge period, various libraries are using Google web advancement purposefully or unwittingly to give organizations. Earlier, if an individual needs to make a record or spreadsheet he was using Microsoft's Office pack, nowadays various libraries are taking care of records, using Google web advancement (Google Docs) on ordinary premise. Usage of Google applications and other equivalent contraptions in the libraries shows an extraordinary move from regular to front line developments. Nowadays, libraries are giving extraordinary number of organizations to their customers to get to various resources and PC applications from a lone stage. This is a favourable situation of disseminated registering. Distributed figuring isn't extraordinarily recognized in open libraries. The clarification behind this is the nonattendance of good master centre in the field of library the officials using pattern setting advancement. Various libraries are thinking to get dispersed figuring anyway they are defying issues in association with organized programming, administrative techniques, spending restrictions, accessibility issue, etc. These days, some remote associations are giving cloud based organizations. Thusly the essential bit of leeway in moving to a dispersed registering condition for a library is the ability to assess new programming without acquiring the hardware similarly as having the alternative to scale the figuring ability to satisfy the need of customers. A library's IT division can be increasingly versatile in raising the proportion of disseminated processing they require by arriving at their dealer of physically getting new hardware, programming and work to meet extended demands. This procedure will put aside the library money and staff resources.

\section{* Working process:-}

Appropriated registering incorporates different cloud parts that talk with each other with the help of use interfaces, generally web organizations. UNIX working system seeks after a similar speculative methodology for its assignments. The endeavour multifaceted nature is disengaged into all of the parts making balanced and sensible results. The two most critical fragments are the back end and the front end. The front end is the interface or the standard screen that is undeniable to the customers and the customers through which they can coordinate with the structure. This interface can be examined with the help of web programs what not the applications can be used with this interface. For the most part this interface relies upon Graphics User

\section{* Interface (GUI).}

The back end incorporates all of the portions and the all out plan and programming arrangement of conveyed figuring, that altogether stays maintained a strategic distance from the customers. Figuratively speaking system perceives what's going on at the back of very customer request. The back end contraption fuses Cloud server, Assistant PCs, Data amassing media and various connectors.

\section{XII.RESULTS}

The result of this paper shows that:-
Choosing distributed cloud computing administration models. These elements are accessible spending plan, versatility to changing client necessities, accessible IT aptitudes, and desperation of required assistance, information security, information protection and unwavering quality of administration. We too proposed a novel multi-criteria approach that takes into thought the total mean of choice qualities and the deviations esteems among the chiefs. In spite of the curiosity of this methodology, it tends to be improved by including more determination criteria, similar to interoperability, execution, adaptability, similarity, multifaceted nature and seller believability and backing.

\section{And also the results show that with the cloud computing} there is:-

$>$ Cost Saving: In distributed computing clients need to pay for the administrations they expended. Upkeep cost is low as client don't have to buy the foundation

$>$ Flexibility: Cloud processing is adaptable. The fast scale all over in the tasks of your business may require snappy alteration of equipment and assets so as to deal with this varieties distributed computing give adaptability.

$>$ Enhanced Security: Cloud registering give high security by utilizing the information encryption, solid access controls, key administration, and security knowledge.

\section{CONCLUSION}

Libraries face mounting challenges in managing the assets of their collection and maintaining or improving service levels to patrons. For the past two decades, libraries have reached out their services to its patrons, initially offering physical forms to adding multimedia items at an advanced level through electronic media. In recent years, impact of cloud computing technology has been tremendous on the library and information centres and the developments are for all to witness. Libraries have been one of the first institutions to be affected by the Information and Communication Technology (ICT) developments and these developments have been exploited effectively to render various information services. Right from the days of designing databases to entering and retrieving records from the database, it has been exciting times for all library professionals. Just when it was time to rejoice the fruits of creating and using local databases on desktops, the communication revolution made its arrival. The cloud computing is lately emerging technology. The basic principle of cloud computing entails the reduction of in-house data centres and the delegation of a portion or all of the Information Technology infrastructure capability to a third party. Universities and Colleges are the core of innovation through their advanced research and development. Subsequently, Higher Institutions may benefit greatly by harnessing the power of cloud computing, including cost cutting as well as all the above types of cloud services. The libraries which do not have sufficient budget to acquire high-end technology with proper hardware and software can choose cloud. 
To cope up with the new technological innovation in the field one needs to know and explore cloud computing. In future, the advantages of this technology may increase its usability, which will relieve libraries and its professionals from the hardships in maintaining the servers, software and manpower.

\section{REFERENCES}

1. Rajkumar Buyya, James Broberg, Andrzej Goscinski: Cloud Computing Principles and Paradigms.

2. S. Ramgovind, M. M. Eloff, and E. Smith, "The management of security in cloud computing," in Information Security for South Africa (ISSA), 2010. IEEE, 2010.

3. F. Sabahi, "Cloud computing security threats and responses," in Communication Software and Networks (ICCSN), 2011 IEEE 3rd International Conference on. IEEE, 2011.

4. Arshdeep Bagha,Vijay Marinetti: Cloud computing: A Hands on Approach.

5. Rackspace.com. The Rack space Cloud. 2012. http://www.rackspace.com/cloud/ (accessed on 01 Aug 2014). 6. Robert Fox, "Library in the Clouds," OCLC Systems \& Services.

6. OCLC. OCLC World share management services.2012

7. Kozokin, Sarit. Ex-Libris cloud: Open for business. 2011.

8. Duracloud.org. Dura cloud. 2012. http://www.duracloud.org, Cloud Computing and Its Application in Libraries.

9. Anthony T.Velte,Toby.J.Velte,Robert: Cloud Computing A practical Approach.

10. Barrie Sonsinsky: Cloud Computing.

11. Robert Fox, "Library in the Clouds," OCLC Systems \& Services

12. Kozokin, Sarit. Ex-Libris cloud: Open for business. 2011.

13. Mitchell, Erik. "Using cloud services for library IT infrastructure." Code4Lib Journal, 2010.

14. Hayes, B. "Cloud computing." Communications of ACM, 2008.

15. M.R.Tribhuwan, V.A.Bhuyar, Shabana Pirzade, "Ensuring Data Storage Security in Cloud Computing through Two-way Handshake based on Token Management", 2010 International Conference on Advances in Recent Technologies in Communication and Computing.

16. Mr. Prashant Rewagad, Ms.Yogita Pawar, "Use of Digital Signature with Diffie Hellman Key Exchange and AES Encryption Algorithm to Enhance Data Security in Cloud Computing”, 2013 International Conference on Communication Systems and Network Technologies.

17. Uma Somani, Kanika Lakhani, Manish Mundra, "Implementing Digital Signature with RSA Encryption Algorithm to Enhance the Data Security of Cloud in Cloud Computing", 1st International Conference on Parallel Distributed and Grid Computing (PDGC 2010). 